\title{
VIENT DE PARAITRE
}

SOULEZ, ANTONIA La Grammaire philosophiquechez Platon. Paris: Presses Universitaires de France, 1991; 348 pp.; ISBN 2-13-043385-5.

"Platon pourrait-il être considéré comme le précurseur de la 'grammaire philoso[hique'-cette 'discipline' à laquelle la philosophie analytique a donné ses titres de noblesse? Cette question, reprise à partir de la tentative méconnue de Gilbert Ryle, dans l'horizon de la philosophie de Russel et du 'second'Wittgenstein, est examinée ici à travers une étude minutieuse de la philosophie du langage du Sophiste et de ses présupposés dans le Cratyle. C'est plus précisément la 'sémantique catégoriale'articulée à la théorie des ldées qui est soumise à l'examen. De la révvaluation de l'outil du langage à la signification des phrases fausses, c'est la voie d'une sémantique de l'énoncé qui s'ouvre sous la forme d'un projet catégorial.

Cette relecture de Platon, en ses acquis et ses limites, permet aussi bien de revenir au coeur même du 'problème' avec lequel s'explique son oeuvre: celui d'une 'grammaire du sens.' Tout se passe comme si le philosophe grec présentait notre distinction moderne entre 'forme logique' et 'forme grammaticale' du langage. Avec les 'genres suprèmes', il s'agit d'une logique non analytique dominée par la figure de l'Autre--ce qui ouvre la voie au 'matheme': question de la liaison de sens que constitue l'énoncé, par où se trouve élaboré un au-delà de la mimesis.

C'est ainsi une oeuvre fondatrice de la philosophie qui est mise en débat avec des schèmes analytiques - au moyen d'une construction qui réfléchit les rapports entre l'oeuvre, la méthode de lecture et la tradition. Ainsi se trouve fixé le seuil où la fondation platonicienne peut faire retour dans la modernité philosophique." 